\title{
Amizade em meninos com Transtorno de Déficit de Atenção/Hiperatividade
}

\author{
Friendship in boys with Attention \\ Deficit/Hyperactivity Disorder
}

\author{
Soraya da Silva SENA \\ Luciana Karine de SOUZA
}

\begin{abstract}
Resumo
Crianças com Transtorno de Déficit de Atenção/Hiperatividade tendem a participar de relações de pares insatisfatórias, podendo ser rejeitadas socialmente e até vitimizadas. O objetivo deste trabalho foi de comparar a percepção da qualidade da melhor amizade e do conflito nessa relação, em 17 meninos com e 19 sem Transtorno de Déficit de Atenção/Hiperatividade, de sete a nove anos de idade, por meio de entrevista semiestruturada e da Escala da Qualidade da Amizade. Os meninos com Transtorno de Déficit de Atenção/Hiperatividade não diferenciaram daqueles sem o transtorno na percepção da qualidade da amizade; destes últimos, os mais velhos pontuaram melhor o amigo. Foi maior a percepção de situações de conflito com o amigo por parte de meninos com Transtorno de Déficit de Atenção/Hiperatividade, inclusive dos mais velhos. É possível que o portador de Transtorno de Déficit de Atenção/Hiperatividade perceba mais os conflitos com o amigo em virtude do aumento etário, pois o avanço sociocognitivo pode auxiliar a captar melhor as reações negativas dos pares a seu comportamento frequentemente desrespeitoso às regras sociais.
\end{abstract}

Unitermos: Criança; Desenvolvimento infantil; Transtorno do déficit de atenção com hiperatividade.

\begin{abstract}
Children with Attention Deficit/Hyperactivity Disorder tend to engage in unsatisfactory peer relationships, being at risk for social rejection or victimization. The aim of this study was to compare the perception of friendship quality and conflict regarding their best friend in 17 boys with and 19 without Attention Deficit/Hyperactivity Disorder. The subjects were 7 to 9 years of age and data collection was performed through a semi-structured interview and application of the Friendship Quality Scale. The perception of friendship quality did not differ between the boys with and without Attention Deficit/Hyperactivity Disorder and the older boys without Attention Deficit/Hyperactivity Disorder scored their perceived friendship quality higher. The perception of conflict situations was higher in boys with Attention Deficit/Hyperactivity Disorder, also higher in those of 9 year of age compared to the younger children with this condition. It is speculated that children with Attention Deficit/Hyperactivity Disorder better perceive conflict situations with a best friend, as they grow older, as social and cognitive growth might help them better capture the negative peer reactions to their frequent disrespect for social rules.
\end{abstract}

Uniterms: Child; Child development; Attetion deficit disorder with hyperactivity disorder.

vr

1 Universidade Federal de Minas Gerais, Faculdade de Filosofia e Ciências Humanas, Departamento de Psicologia. Av. Antônio Carlos, 6627, Sala F-4050, Campus Pampulha, 31270-901, Belo Horizonte, MG, Brasil. Correspondência para/Correspondence to: L.K. SOUZA. E-mail: <lucianak@fafich.ufmg.br>. Artigo elaborado a partir da dissertação de S.S. SENA, intitulada "Amizade em meninos com TDAH". Universidade Federal de Minas Gerais, 2009.

Agradecimentos: V. Haase, L. Magalhães, T. Carvalho, S. Peron, G. Gauer e às crianças e seus familiares. 
A amizade em crianças é um tema caro especialmente a cientistas da área das humanidades há aproximadamente um século. Na psicologia, a amizade infantil é valorizada em teorias psicológicas clássicas como a de Jean Piaget, e seu foco no desenvolvimento sociocognitivo a partir das relações de pares, bem como em modelos mais atuais como o de Robert Selman, que destacou o papel das amizades no desenvolvimento da habilidade de troca de papéis e empatia.

A amizade tem o potencial de promover desenvolvimento, aprendizagem e saúde, o que vem sendo argumentado por pesquisadores e observado em estudos empíricos. Trata-se de uma relação social que permite o avanço de aspectos como a confiança, a lealdade, a empatia, a generosidade, a oferta de apoio (emocional ou instrumental, concreto), a proximidade e o companheirismo (Hartup \& Stevens, 1997; Newcomb \& Bagwell, 1995). Difere em suas características ao longo da infância, acompanhando o desenvolvimento cognitivo e socioemocional da criança, com reflexos na compreensão da amizade e no comportamento e atitudes direcionados aos amigos (Antoniazzi et al., 2001; Benenson, 1993; Buhrmester, 1996; Bukowski, Newcomb \& Hartup, 1996; Newcomb \& Bagwell, 1995).

No Brasil, já não cabe dizer que a produção científica sobre amizade em crianças é escassa. O próprio investimento científico no tema da amizade, em qualquer faixa etária e em diversos contextos, vem recebendo destaque e interesse por pesquisadores de todo o País (Garcia, 2010; Souza \& Hutz, 2012).

A amizade diante de transtornos na infância, por outro lado, ainda é pouco estudada no Brasil. Os estudos publicados disponíveis analisaram as relações de amizade em crianças com diagnósticos como autismo, hiperatividade, Síndrome de Willis e Síndrome de Down (Albertassi \& Garcia, 2006), diabetes e câncer (Ferreira \& Garcia, 2008). Apenas dois trabalhos brasileiros trataram da desatenção e do Transtorno de Déficit de Atenção/Hiperatividade (TDAH) na interface com as amizades infantis.

Tonelotto (2002) identificou alunos desatentos no ambiente escolar, investigando sua percepção e a dos colegas quanto à popularidade e à rejeição social na sala de aula. As crianças com problemas de atenção demonstraram mais comportamentos negativos em relação à escola, e mais atitudes negativas sobre seus pares, que, por sua vez, também perceberam essas crianças desatentas de modo mais negativo. A impopularidade foi mais observada nas crianças com problemas de atenção, que foram também mais citadas como rejeitadas e menos citadas como aceitas pelos colegas. Parece importante destacar a escola como promotora da socialização infantil e de habilidades sociais; no entanto, seu foco nas habilidades acadêmicas tem dado papel secundário ao desenvolvimento de habilidades sociais entre os alunos (Z. Del Prette \& A. Del Prette, 2003).

Albertassi e Garcia (2006) relatam as relações de amizade de um garoto hiperativo, de dez anos de idade, que possuía um melhor amigo que o acompanhava em todas as atividades escolares. Os colegas de aula nomeados como amigos pelo garoto também o nomearam como amigo, embora os professores tenham apontado que ele não compartilhava interesses com o grupo, permanecendo com os colegas somente se a atividade fosse de seu interesse. Os pais e professores do menino avaliavam suas amizades como restritas e superficiais, mesmo reconhecendo seu carinho e fidelidade aos amigos. Segundo Albertassi e Garcia (2006), o padrão de interação do garoto com seus amigos foi similar ao de crianças sem desatenção. Portanto, são realmente necessárias mais pesquisas sobre as amizades de crianças com TDAH, especialmente com amostra maior.

OTDAHé uma síndrome neurocomportamental caracterizada por um padrão persistente de desatenção e/ou hiperatividade-impulsividade (American Psychiatric Association, 2002; Rotta, 2006). Trata-se de um transtorno marcado por um nível inadequado de atenção para o que é esperado para a idade, posicionando-o como transtorno do desenvolvimento. É dividido em três tipos: desatento, hiperativo-impulsivo e combinado. Carrega déficits motores, perceptivos, cognitivos e comportamentais (Barkley, 1997, 2002; Rotta, 2006). É na esfera das dificuldades comportamentais que as relações de amizade da criança com TDAH serão foco de investigação e intervenção neste artigo.

Em recente oportunidade foi discutido o fenômeno social da amizade em crianças com TDAH (Sena \& Souza, 2010). De acordo com a literatura especializada, as crianças com TDAH tendem a participar de relações 
de pares insatisfatórias e deterioradas, não raro envolvendo-se em situações nas quais são rejeitadas socialmente e até vitimizadas (Desidério \& Miyazaki, 2007; Hoza et al., 2005; Pelham Jr \& Bender, 1982; Tonelotto, 2002; Unnever \& Cornell, 2003). Segundo S. Goldstein e M. Goldstein (2002), os colegas destas crianças demonstram reações autoritárias e controladoras em resposta ao comportamento disruptivo, desatento, imaturo e provocativo das crianças com TDAH. Além disso, o portador deste transtorno costuma falar em demasia, interage menos com as outras crianças e é menos apto a cooperar, dividir e manter promessas (Barkley, 2002). Também tendem a ser mais "mandões" diante da rejeição social, o que a retroalimenta (S. Goldstein \& M. Goldstein, 2002; Phelan, 2005). Estando alterados, nestes casos, o adiamento de gratificações, o controle de impulsos, a hiperatividade, a hiper-responsividade e o respeito a regras, fica dificultada a vida social da criança com TDAH, especialmente fazer e manter amizades (Barkley, 2002; S. Goldstein \& M. Goldstein, 2002; Phelan, 2005).

Em um nível mais superficial de relacionamentos a criança com TDAH exerce atração sobre as outras crianças dada suas usuais características de dinamismo, diversão e disposição para aventuras. No TDAH de tipo desatento, o portador comete deslizes que divertem seus pares, como usar sapatos trocados, inverter nomes e esquecer objetos. Para uma amizade mais próxima e íntima, porém, a criança com TDAH pode trazer problemas e decepções. São obstáculos à vida social seus comportamentos de "furar fila", "dizer tudo que vem à cabeça", perder brinquedos e não perceber as sutilezas não verbais nas brincadeiras e conversas com as outras crianças. Apesar disso, os amigos do portador de TDAH podem fornecer apoio no enfrentamento de dificuldades e desafios, pois isso é esperado de boas amizades. Com ajuda apropriada, a criança com TDAH pode ser autêntica no relacionamento com uma amizade verdadeira (Sena \& Diniz Neto, 2005).

Diante dos dados disponíveis na literatura científica sobre as relações de amizade de crianças com TDAH, em especial, a escassez de estudos deste tipo, acredita-se ser relevante investigar como a criança com TDAH difere daquela sem este diagnóstico na percepção de suas amizades. Em virtude do maior índice de meninos com TDAH, estes foram o foco do trabalho.
Ademais, dada a indicação da literatura sobre dificuldades do portador nas relações com amigos, a percepção sobre situações de conflito com a melhor amizade também será abordada no texto. Assim, o objetivo do trabalho foi comparar a percepção da qualidade da melhor amizade e a percepção de conflito nessa relação em meninos com e sem TDAH, de sete a nove anos de idade.

\section{Método}

Participaram do estudo 18 crianças com TDAH, que constituíram o Grupo Clínico (GC), e 21 sem TDAH, que compuseram o Grupo Típico (GT), do sexo masculino, com idade entre sete e nove anos, residentes na Região Metropolitana de Belo Horizonte (MG). Buscou-se pareamento etário entre o GC e o GT (meninos de sete, de oito, e de nove anos) em virtude de possíveis diferenças etárias nas amizades infantis (Antoniazzi et al., 2001; Benenson, 1993; Buhrmester, 1996; Bukowski et al., 1996; Newcomb \& Bagwell, 1995). A opção por exclusividade do sexo masculino deveu-se ao maior acesso a esse grupo de portadores de TDAH nos serviços de saúde (Gaião e Barbosa, 2003). A idade mínima segue a indicação do Diagnostic and Statistic Manual of Mental Disorders - Fourth Edition - Text Revision (DSM-IV-TR) (American Psychiatric Association, 2002), e a máxima deve-se à ocorrência de mudança sintomática com a entrada na puberdade (Prado \& Bromberg, 2005).

A média de idade do GT foi de oito anos e seis meses. A média de idade do GC foi de oito anos e quatro meses. Destes 18 meninos, 78\% vinham sendo medicados, na época da pesquisa, há pelo menos três meses, e $72 \%$ deles estavam participando de pelo menos um outro tratamento para o TDAH.

As crianças do GC foram indicadas por um ambulatório de hospital universitário, por uma escola estadual e por um profissional da psicologia que atende meninos com TDAH. O GT foi proveniente de uma escola pública. As instituições foram escolhidas por conveniência, sendo observada a similaridade socioeconômica entre o GC e o GT (nível socioeconômico médio, conforme referido pelas instituições). Abordaram-se, ainda, 23 professores das crianças participantes. Esses professores foram convidados a responder 
à Escala de Avaliação do Comportamento Infantil para o Professor (EACl-P) (Brito, 2006) em virtude da necessidade de exclusão de crianças com problemas comportamentais graves e/ou transtornos mentais porventura presentes no GT.

Foram realizadas entrevistas individuais semiestruturadas e a aplicação da Escala sobre Qualidade da Amizade (EQA) (Lisboa, 2005). A entrevista foi inspirada em Antoniazzi et al. (2001) e em estudos empíricos salientes na literatura científica sobre amizade infantil (por exemplo, número de amigos, presença de melhor amizade). Nos meninos com TDAH, buscou-se saber também a presença de comorbidade, o tempo de uso de medicamentos e a presença de outros tratamentos concomitantes, variáveis estas que podem exercer influências nas relações de amizade de portadores de TDAH (Frankel \& Feinberg, 2002; Hoza, Mrug, Pelham Jr., Greiner \& Gnagy, 2003). As crianças do GT obtiveram resultados individuais apropriados nas EACI-P preenchidas por seus professores; no entanto, de 18 meninos com TDAH, para apenas três deles obteve-se o preenchimento da EACI-P.

A Escala Sobre Qualidade da Amizade é um instrumento de avaliação da qualidade da melhor amizade de crianças e adolescentes (Bukowski, Hoza \& Boivin, 1994) adaptada por Lisboa (2005). Essa escala é composta por 22 frases sobre as quais a criança afirma concordância ou discordância com o que está escrito, desde Discordo Totalmente (opção 1) até Concordo Totalmente (opção 5). As frases da escala avaliam, em cinco subescalas, aspectos fundamentais da amizade: Companhia, Conflito, Apoio, Segurança e Intimidade (Bukowski et al., 1994). O máximo de pontos que se pode obter na EQA é de 110. À exceção das subescalas Companhia e Conflito, todas as outras demonstraram consistência interna adequada (Lisboa, 2005). O uso da EQA no trabalho que ora se descreve foi consentido pela autora.

Após a obtenção do consentimento formal assinado por um dos responsáveis de cada criança, foi pedido, ainda, à própria criança seu consentimento para participação na pesquisa, respeitando-se a vontade livre da mesma. As entrevistas, gravadas e transcritas no caso do GT, e o preenchimento da EQA foram realizados nas dependências das instituições colaboradoras ou nas 332 residências das crianças (no caso do GC). O gravador não foi utilizado com o GC porque no estudo-piloto o mesmo era um fator de distração para estes meninos. O estudo-piloto também evidenciou que as crianças com idade menor às do trabalho de Lisboa (2005) não demonstraram dificuldades em responder a EQA. O estudo foi aprovado pelo Comitê de Ética em Pesquisa da Universidade Federal de Minas Gerais em 10/6/2008 (Protocolo no 135/08). Todos os participantes assinaram um Termo de Consentimento Livre e Esclarecido antes de sua inclusão na amostra.

Foram calculados os escores totais na EQA para os dois grupos (GT e GC) seguindo o critério de correção adotado por Lisboa (2005). O GC obteve média de 91,56 pontos, enquanto o GT obteve 84,57 pontos. Porém, notou-se que as pontuações obtidas por três participantes (um do GC e dois do GT) desviavam-se acentuadamente dos valores médios de seus grupos; essas três crianças foram excluídas da amostra como outliers.

O cálculo das médias de pontos na EQA foi, então, realizado com dados de 17 meninos do GC e 19 meninos do GT. Além disso, a subescala Conflito da EQA foi analisada separadamente em virtude da indicação dada pela literatura científica de que crianças com TDAH envolvem-se em mais conflitos interpessoais com pares. Foram utilizados testes $t$ para amostras independentes, e os testes não paramétricos Mann-Whitney e KruskalWallis. Este último foi utilizado para comparar as médias da EQA e da subescala Conflito nas três faixas de idade do GT e do GC. Cabe lembrar que o teste Kruskal-Wallis não utiliza os escores, mas os postos médios dos escores, e seu resultado é expresso por meio de um Qui-quadrado. Todas as análises estatísticas foram feitas utilizando-se o pacote estatístico SPSS 17.0.0, com nível de significância a 5\%.

\section{Resultados}

Todos os meninos do estudo, tanto do GT como do GC, indicaram a existência de, no mínimo, um amigo. Todos também apontaram a existência de uma melhor amizade.

Com relação à percepção da qualidade da melhor amizade, na EQA a média do GT ficou em 88,42 $(\mathrm{DP}=10,11)(\mathrm{n}=19)$, enquanto a média do GC foi de 93,76 $(D P=8,31)(n=17)$. O teste $t$ não indicou diferença 
significativa entre o GC e o GT na EQA ( $\mathrm{t}=-1,72$; gl=34; $p=0,095)$; tampouco o teste Mann-Whitney ( $U=109,5$; $z=-1,653 ; p=0,100$; one-tailed). Desta forma, a avaliação propiciada pela EQA sugere uma similaridade entre a percepção dos meninos do GT e dos meninos do GC sobre a qualidade da amizade com o melhor amigo.

Quanto à percepção dos meninos sobre conflitos com o melhor amigo, a média de pontos na subescala Conflito do GC foi de 15,88 (DP=4,13) ( $n=17)$, enquanto no $\mathrm{GT}$ foi de $12,58(\mathrm{DP}=3,83)(\mathrm{n}=19)$. O teste $t$ mostrou diferença significativa $(t=-2,48$; $g l=34$; $p=0,018)$, bem como o teste Mann-Whitney ( $U=91,00$; $z=-2,268 ; p=0,025$; one-tailed). Portanto, os meninos com TDAH deste estudo perceberam mais as situações de conflito com a melhor amizade, se comparados com os meninos sem TDAH.

Também foi objetivo da pesquisa conferir a percepção dos meninos sobre a qualidade da melhor amizade com base em três faixas de idade. No GT, as médias e desvios-padrão encontrados para a EQA foram os seguintes: para os meninos com sete anos de idade $(n=5)$ a média foi de 85,00 (DP=6,92); para oito anos $(n=7)$ foi de 83,57 (DP=12,66); e para nove anos $(n=7)$ foi de 95,71 ( $D P=3,90)$. O teste Kruskal-Wallis mostrou diferença significativa $\left(\chi^{2}=7,117 ; g \mathrm{l}=2 ; p=0,028\right)$ entre os postos médios das três faixas de idade: 6,50 para sete anos, 8,07 para oito, e 14,43 para nove anos. Este resultado sugere um aumento na percepção da qualidade da amizade com o avanço etário nos meninos sem TDAH. Já no GC, a média dos meninos com sete anos de idade ( $n=2)$ foi de $93,00(\mathrm{DP}=1,41)$; com oito anos ( $n=8$ ) foi de 90,12 (DP=9,83); e com nove anos ( $n=7$ ) foi de 98,14 (DP=5,55). O teste Kruskal-Wallis não indicou diferença significativa $\left(\chi^{2}=3,857 ; g l=2 ; p=0,145\right)$, com média de 7,00 tanto para sete anos como para oito anos, e média de 11,86 para os meninos de nove anos com TDAH.

Foram calculadas as médias do GT e do GC na subescala Conflito nas três faixas de idade. No GT, a média dos meninos de sete anos foi de 12,4 ( $\mathrm{DP}=4,09)$; dos de oito anos foi de 11,85 (DP=4,09); e dos de nove anos foi de 13,42 (DP=3,82). O teste Kruskal-Wallis não apontou diferença significativa entre as faixas de idade do $\mathrm{GT}\left(\chi^{2}=0,564 ; \mathrm{gl}=2 ; p=0,754\right)$, e os postos médios foram de 9,60 para sete anos, 9,07 para oito anos e 11,21 para nove anos. No GC, os meninos de sete anos apre- sentaram média de 8,00 na subescala Conflito ( $D P=5,65)$; nos de oito anos a média foi de 16,12 $(\mathrm{DP}=2,74)$; e nos de nove anos foi de 17,85 ( $\mathrm{DP}=2,47)$. O teste Kruskal-Wallis apontou diferença significativa entre os postos médios das três faixas etárias $\left(\chi^{2}=5,975\right.$; $g \mid=2 ; p=0,050)$, que foram 1,75 para sete anos, 8,75 para oito anos, e 11,36 para nove anos de idade nos meninos com TDAH. Esses resultados segurem que no grupo de crianças com TDAH a percepção do conflito com a melhor amizade aumenta com a idade.

\section{Discussão}

Os resultados apontaram para uma realidade diversa do que é comumente divulgado na literatura sobre as relações interpessoais de crianças com TDAH. As crianças com TDAH teriam poucos amigos (ou nenhum), ou então a percepção de tais crianças sobre suas relações interpessoais estaria alterada, ou seja, elas não perceberiam suas dificuldades interpessoais e teriam critérios menos rigorosos de avaliação de quem seja seu amigo. Parte da literatura indica maior probabilidade de esta última hipótese estar correta (Barkley, 2002; Diamantopoulou, Henricsson \& Rydell, 2005; Pelham Jr \& Bender, 1982).

Contrariando a literatura, os 39 meninos do estudo realizado indicaram no mínimo um amigo e também uma melhor amizade. Tendo como referência a pesquisa de Albertassi e Garcia (2006), pode-se dizer que a pesquisa aqui descrita confirma dados da literatura brasileira sobre a percepção da amizade em meninos com TDAH. Em outras palavras, crianças portadoras deste transtorno percebem amigos dentre seus pares. Além disso, não foi detectada diferença significativa na percepção da qualidade da melhor amizade (escore da EQA) em meninos com TDAH e sem TDAH. Assim, esses resultados não parecem ir na mesma direção daqueles provenientes de estudos que acusam ausência de amizades e/ou uma percepção distorcida da criança com TDAH sobre seus amigos (Barkley, 2002; Desidério \& Miyazaki, 2007; Diamantopoulou et al., 2005; Hoza et al., 2005; Pelham Jr \& Bender, 1982; Tonelotto, 2002; Unnever \& Cornell, 2003).

Especificamente com respeito aos meninos sem TDAH, detectou-se diferença significativa na percepção da qualidade da melhor amizade nas três faixas de idade 
estudadas. É possível considerar que o avanço etário tenha influenciado positivamente a percepção da qualidade da melhor amizade em meninos típicos entre os sete e nove anos de idade. Esse dado pode ser explicado pela maior capacidade cognitiva experimentada pelas crianças com o avanço da idade: ao se desenvolverem, as crianças se tornam mais capazes de perceber as questões envolvidas nas relações de amizade porque ganham habilidades intelectuais importantes que as auxiliam no convívio social (Piaget, 1932/1977; Selman \& Byrne, 1974).

Os meninos com TDAH se destacaram dos meninos típicos com escores maiores na percepção de situações de conflito com a melhor amizade. A literatura científica confirma a presença de relações interpessoais conflituosas na vida social da criança portadora de TDAH (Barkley, 2002; S. Goldstein \& M. Goldstein, 2002; Phelan, 2005); no entanto, o que o resultado sugere é que o menino portador de TDAH está atento a uma importante dimensão das relações de amizade: o conflito. Ainda assim, não se pode afirmar com clareza, na comparação com o menino típico, que o portador está percebendo mais as situações de conflito com o melhor amigo porque tende a não lidar bem com crianças que discordam de sua vontade, o que o coloca diante de uma maior quantidade de situações nas quais os amigos reagem negativamente a ele. Ademais, futuros estudos com maior controle de variáveis e amostra maior podem investigar melhor a relação entre ser portador de TDAH, perceber determinadas situações com o melhor amigo como situações conflituosas, acessar a percepção do melhor amigo sobre estas mesmas situações, bem como outros aspectos carentes de pesquisa dentro do escopo das relações sociais de crianças com TDAH.

O que futuros estudos também precisarão investigar é a possibilidade da interferência de comorbidades nos meninos do GC. Meninos com diagnóstico de TDAH, mas sem comorbidades, podem demonstrar percepção diferente sobre seu melhor amigo.

Ao verificar a influência da idade na percepção do conflito com a melhor amizade em meninos sem TDAH, não foi encontrada diferença significativa. Entre-

334 tanto, o mesmo não se verificou em meninos com
TDAH. Os resultados sugerem que o menino com TDAH aos nove anos de idade é mais capaz de perceber as situações de conflito com a melhor amizade do que o menino com TDAH aos sete ou oito anos de idade, ou seja, à medida que avança em seu desenvolvimento sociocognitivo. O avanço sociocognitivo pode capacitar a criança portadora a captar melhor as reações negativas dos pares a seu comportamento frequentemente desrespeitoso às regras sociais. Todavia, mesmo que a criança com TDAH de mais idade demonstre perceber melhor os conflitos presentes na relação com seus amigos, ela carece de comportamentos mais adequados para lidar com estas situações.

Também é lícito considerar que o TDAH ressalta, nas crianças amigas da criança portadora, os conflitos vividos entre eles; além disso, o avanço cognitivo-evolutivo colabora para a capacidade de melhor perceber estes conflitos. Por exemplo, provavelmente a criança típica de nove anos que interage com a portadora de TDAH de mesma idade fornece um feedback mais claro sobre os comportamentos e atitudes desta última, dando mais destaque às situações de conflito quando elas ocorrem, na comparação com crianças mais novas portadoras de TDAH e seus pares de mesma faixa etária. Sobre estes aspectos, e com inspiração nos trabalhos de Daudt, Souza e Sperb (2007a e b), sugere-se uma pesquisa sobre amizade infantil com díades de crianças com e sem TDAH, amigas e não amigas.

As amizades têm sido descritas na literatura científica como fator de proteção contra a vitimização (Lisboa \& Koller, 2001; Rubin \& Coplan, 1992). Há, a título de exemplo, trabalhos que evidenciam que o comportamento pró-social de amigos reduz o risco de crianças mais vulneráveis serem alvo de agressão por pares (Lamarche et al., 2006). Embora não tenha sido objetivo deste estudo, houve relato desse comportamento protetor por parte de uma criança com TDAH. O participante Q do GC assim referiu seu melhor amigo: " $a$ gente) brinca, ele me defende...", "ele me chama de filho". Portanto, pode-se perceber na fala desse entrevistado a função protetora da amizade contra a agressão por pares, além do entretenimento por meio de brincadeiras. Todavia, pesquisas focalizadas nestas questões e com metodologia predominantemente qualitativa poderão avaliar mais detalhadamente a relação entre amizade infantil, TDAH e agressão por pares. 


\section{Considerações Finais}

A principal limitação encontrada na investigação realizada foi o número reduzido de participantes, especialmente meninos com TDAH aos sete anos de idade. Portanto, os resultados obtidos não permitem generalizações. Para futuros estudos poderá ser mais proveitosa a realização de pesquisas sobre as amizades de portadores de TDAH com no mínimo oito anos de idade.

Além disso, pesquisar as relações de amizade em portadores de TDAH na adolescência pode ser de grande riqueza, dado que, nesta fase do desenvolvimento, as amizades ocupam um espaço privilegiado, em detrimento do ambiente familiar. E, uma vez que se sugere maior abrangência etária na pesquisa da amizade em portadores de TDAH, recomenda-se também a realização do estudo com portadores adultos, inclusive no que diz respeito aos seus conceitos de amizade e a reciprocidade real (e não somente relatada) destas amizades.

A questão da inclusão de portadores de TDAH do sexo feminino na pesquisa das relações de amizade é relevante e poderia vislumbrar dados importantes sobre esse relacionamento interpessoal. É uma questão, porém, desafiadora em termos de pesquisa científica, dado que há menos portadores de TDAH do sexo feminino que do sexo masculino, mas poderia disponibilizar conhecimento sobre o relacionamento de amizade em meninas e/ou mulheres portadoras de TDAH.

Com relação ao uso da EACl-P em pesquisas futuras, há que se considerar a necessidade de cuidado ao abordar os professores. Contato somente por escrito mostra-se pouco eficaz, bem como a ausência de indicação de prazo para devolução de instrumentos preenchidos. Portanto, sugere-se, na impossibilidade de contato pessoal, no mínimo um contato telefônico com o professor respondente. Ademais, o trabalho com professores sobre o TDAH é desejável, especialmente quando no contexto escolar há comportamentos infantis vistos por professores como indícios de hiperatividade e associados a um suposto diagnóstico de TDAH. Oficinas com professores podem contribuir nessa direção, a exemplo do trabalho realizado por Carvalho, Peron, Sena e Souza (2009).
A pesquisa das relações interpessoais em portadores de TDAH é um campo de trabalho desafiador e digno de esforços futuros Requer o conhecimento da produção científica atual sobre este transtorno nos vários aspectos que o compõem (seu histórico, as definições mais usadas, a etiologia e prevalência, o diagnóstico e tratamento, entre outros). É um empenho recompensador pela contribuição científica trazida e, principalmente, pela possibilidade de reflexão sobre uma relação interpessoal tão rica e prazerosa como é a amizade.

\section{Referências}

Albertassi, I., \& Garcia, A. (2006). Crianças com necessidades especiais e seus amigos: um estudo na cidade de Vitória (ES). In A. Garcia (Org.), Relacionamento interpessoal: estudos e pesquisas (pp.55-73). Vitória: UFES.

American Psychiatric Association. (2002). DSM-IV-TR: manual diagnóstico e estatístico de transtornos mentais (4a ed.). Porto Alegre: Artmed

Antoniazzi, A., Hutz, C. S., Lisboa, C., Xavier, C., Eickhoff, F., \& Bredemeier, J. (2001). O desenvolvimento do conceito de amigo e inimigo em crianças e pré-escolares. Psico-USF, 6(2), 1-9.

Barkley, R. A. (1997). Behavioral inhibition, sustained attention, and executive functions: Constructing a unifying theory of ADHD. Psychological Bulletin, 121(1), 65-94.

Barkley, R. A. (2002). Transtorno do déficit de atenção/hiperatividade: guia completo parapais, professores eprofissionais de saúde. Porto Alegre: Artmed.

Benenson, J. (1993). Greater preference among females than males for dyadic interaction in early childhood. Child Development, 64(2), 544-55.

Brito, G. (2006). EACl-P:escala de avaliação do comportamento infantil para o professor. São Paulo: Vetor.

Buhmester, D. (1996). Need fulfillment, interpersonal competence, and the developmental contexts of early adolescent friendship. In W. Bukowski, A. Newcomb \& W. Hartup (Orgs.), The company they keep: Friendship in childhood and adolescence (pp.158-185). Cambridge: University Press.

Bukowski, W., Hoza, B., \& Boivin, M. (1994). Measuring friendship quality during pre- and early adolescence: The development and psychometric properties of the friendship qualities scale. Journal of the Social and Personal Relationships, 11(3), 471-84.

Bukowski, W., Newcomb, A., \& Hartup, W. (1996). Friendship and its significance in childhood and adolescence: Introduction and comment. In W. Bukowski, A. Newcomb \& W. Hartup (Orgs.), The company they keep: Friendship in childhood and adolescence (pp.1-15). Cambridge: University Press. 
Carvalho, T. L., Peron, S. I., Sena, S. S., \& Souza, L. K. (2009). Transtorno de déficit de atenção/hiperatividade na interface saúde e educação: uma experiência com educadores. Revista Pedagógica, 11(23), 283-294.

Daudt, P., Souza, L. K., \& Sperb, T. (2007a). Amizade e gênero nos conflitos de pré-escolares. Interpersona, 1(1), 1-19.

Daudt, P., Souza, L. K., \& Sperb, T. (2007b). Amizade na criança pequena: padrões de interação e diferenças de gênero. In L. K. Souza \& C. S. Hutz (Orgs.), Amizade em contexto: desenvolvimento e cultura. São Paulo: Casa do Psicólogo.

Del Prette, Z., \& Del Prette, A. (2003). Habilidades sociais e educação: pesquisa e atuação em psicologia escolar/ educacional. In Z. Del Prette (Org.), Psicologia escolar e educacional: saúde e qualidade de vida (pp.113-41). Campinas: Alínea.

Desidério, R., \& Miyazaki, M. (2007). Transtorno de déficit de atenção/hiperatividade: orientações para a família. Psicologia Escolar e Educacional, 11(1), 165-176.

Diamantopoulou, S., Henricsson, L., \& Rydell, A. (2005). ADHD symptoms and peer relations of children in a community sample: Examining associated problems, self-perceptions, and gender differences. International Journal of Behavioral Development, 29(5), 388-398.

Ferreira, B., \& Garcia, A. (2008). Aspectos da amizade de adolescentes com diabetes e câncer. Estudos de Psicologia (Campinas), 25(2), 293-301. doi:10.1590/S0103-166X200 8000200013.

Frankel, F., \& Feinberg, D. (2002). Social problems associated with ADHD vs. ODD in children referred for friendship problems. Child Psychiatry and Human Development, 33(2), 125-46.

Gaião e Barbosa, A.A. (2003). O TDAH em meninas: características especiais? In L. Rohde \& P. Mattos (Orgs.), Princípios e práticas em TDAH (pp.143-149). Porto Alegre: Artmed.

Garcia, A. (Org.). (2010). Relacionamento interpessoal: uma perspectiva interdisciplinar. Vitória: ABPRI.

Goldstein, S., \& Goldstein, M. (2002). Seriam os amigos um sonho impossível para a criança hiperativa? In S. Goldstein \& M. Goldstein. Hiperatividade: como desenvolver a capacidade de atenção da criança (pp.81-104). Campinas: Papirus.

Hartup, W., \& Stevens, N. (1997). Friendships and adaptation in the life course. Psychological Bulletin, 121(3), 355-70.

Hoza, B., Mrug, S., Gerdes, A., Bukowski, W., Kraemer, H., Wigal, T., et al. (2005). What aspects of peer relationships are impaired in children with attention deficit hyperactivity disorder? Journal of Consulting and Clinical Psychology, 73(3), 411-23.

Hoza, B., Mrug, S., Pelham Jr., W., Greiner, A., \& Gnagy, E. (2003). A friendship intervention for children with attention-deficit/hyperactivity disorder: Preliminary findings. Journal of Attention Disorders, 6(3), 87-96.

Lamarche, V., Brendgen, M., Boivin, M., Vitaro, F., Pérusse, D., \& Dionne, G. (2006). Do friendships and sibling relationships provide protection against peer victimization in a similar way? Social Development, 15(3), 373-93.

Lisboa, C. S. M. (2005). Comportamento agressivo, vitimização e relações de amizade de crianças em idade escolar: fatores de risco e proteção (Tese de doutorado não-publicada). Instituto de Psicologia, Universidade Federal do Rio Grande do Sul.

Lisboa, C. S. M., \& Koller, S. H. (2001). Considerações éticas na pesquisa e na intervenção sobre violência doméstica. In C. S. Hutz (Org.), Situações de risco e vulnerabilidade na infância e na adolescência: aspectos teóricos e estratégias de intervenção (pp.187-212). São Paulo: Casa do Psicólogo.

Newcomb, A., \& Bagwell, C. (1995). Children's friendship relations: A meta-analytic review. Psychological Bulletin, 117(2), 306-47.

Pelham Jr., W., \& Bender, M. (1982). Peer relationships in hyperactive children: description and treatment. Advances in Learning and Behavioral Disabilities, 1, 366-436.

Phelan, T. (2005). TDA/TDAH: Transtorno de déficit de atenção e hiperatividade. São Paulo: Makron Books.

Piaget, J. (1977). O julgamento moral da criança. São Paulo: Mestre Jou. (Originalmente publicado em 1932).

Prado, H., \& Bromberg, M. (2005). O adolescente e o TDA/H. In A. Hounie \& W. Camargos Jr. (Orgs.), Manual clínico do transtorno de déficit de atenção/hiperatividade (pp.480505). Belo Horizonte: Info.

Rotta, N. T. (2006). Transtorno da atenção: aspectos clínicos. In N. T. Rotta, L. Ohlweiler \& R. Riesgo (Orgs.), Transtornos da aprendizagem: abordagem neurobiológica e multidisciplinar (pp.301-13). Porto Alegre: Artmed.

Rubin, K., \& Coplan, R. (1992). Peer relationships in childhood. In M. Bornstein \& M. Lamb (Orgs.), Developmental psychology: An advanced textbook (pp.519-78). Hillsdale: Lawrence Erlbaum.

Selman, R., \& Byrne, D. (1974). A structural-developmental analysis of levels of role taking in middle childhood. Child Development, 45(3), 803-806.

Sena, S. S., \& Souza, L. K. (2010). Amizade, infância e TDAH. Contextos Clínicos, 3(1), 18-28.

Sena, S. S., \& Diniz Neto, O. (2005). Distraído e a 1000 por hora: perguntas e respostas sobre transtorno de déficit de atenção e hiperatividade. Belo Horizonte: Anomelivros.

Souza, L. K., \& Hutz, C. S. (2012). Amizade em contexto: desenvolvimento e cultura. São Paulo: Casa do Psicólogo.

Tonelotto, J. (2002). Aceitação e rejeição: percepção de escolares desatentos no ambiente escolar. Psicologia Escolar e Educacional, 6(2), 141-148.

Unnever, J., \& Cornell, D. (2003). Bullying, self-control, and ADHD. Journal of Interpersonal Violence, 17(2), 129-147.

Recebido em: 28/4/2011

Versão final em: 26/3/2012

Aprovado em: 7/5/2012 